\title{
Vibration based electromagnetic micropower generator on silicon
}

\author{
Santosh Kulkarni, Saibal Roy, ${ }^{\text {a) }}$ and Terence O’Donnell \\ Tyndall National Institute, Cork, Ireland \\ Steve Beeby and John Tudor \\ University of Southampton, Southampton, United Kingdom
}

(Presented on 3 November 2005; published online 27 April 2006)

\begin{abstract}
This paper discusses the theory, design and simulation of electromagnetic micropower generators with electroplated micromagnets. The power generators are fabricated using standard microelectromechanical system processing techniques. Electromagnetic two-dimensional finite element anlysis simulations are used to determine voltage and power that can be generated from different designs. This paper reports a maximum voltage and power of $55 \mathrm{mV}$ and $70 \mu \mathrm{W}$ for the first design, incorporating microfabricated two-layer $\mathrm{Cu}$ coils on a $\mathrm{Si}$ paddle vibrating between two sets of oppositely polarized electroplated $\mathrm{Co}_{50} \mathrm{Pt}_{50}$ face centered tetragonal phase hard magnets. A peak voltage and power of $950 \mathrm{mV}$ and $85 \mu \mathrm{W}$ are obtained for the second design, which includes electroplated $\mathrm{Ni}_{45} \mathrm{Fe}_{55}$ as a soft magnetic layer underneath the hard magnets. The volume of the device is about $30 \mathrm{~mm}^{3}$. (C) 2006 American Institute of Physics. [DOI: 10.1063/1.2176089]
\end{abstract}

Recent advances in the design of low power very large scale integrated (VLSI) circuits ${ }^{1,2}$ along with low duty cycles of wireless sensors have reduced the power requirement for integrated nodes from the milliwatt to the microwatt range. This enabled researchers to explore the possibility of scavenging energy from the local environment as an alternative to batteries. Lately, harvesting energy from vibrations has become the focus of intense research mainly for powering different autonomous sensor nodes to augment their lifetimes. Electromagnetic power generators have an advantage of providing potentially infinite amount of energy, without depending on intrinsic coupling factor as in piezoelectric generator. ${ }^{3}$ In a recent work, ${ }^{4}$ a $10 \mathrm{~mm}$ wide microgenerator has been developed with a spinning magnet above a mesh of coils fabricated on a chip. To date, there has not been any report of electromagnetic power generators microfabricated using electroplated micromagnets.

Previous work has demonstrated electromagnetic power generators using fixed bulk magnets such as $\mathrm{NdFeB}$ and a moving $\mathrm{Cu}$ coil attached to a stainless steel cantilever beam. ${ }^{5}$ This generator having a size of $3000 \mathrm{~mm}^{3}$ was tested on a car engine and produced a peak power of $3.9 \mathrm{~mW}$. In other work, ${ }^{6}$ a generator with the magnets located on a stainless steel beam and vibrating around a coil is described. This design produced a constant power of $2.5 \mathrm{~mW}$, with a load resistance of $100 \Omega$. In one of our recent works, ${ }^{7}$ the voltage and power outputs have been measured in the range of $0.7-4.15 \mathrm{~V}$ and $104-122 \mathrm{nW}$ across a $100 \Omega$ load resistance with a wire-wound coil integrated into a silicon paddle vibrating between two sets of bulk NdFeB magnets. The volume of the device was approximately $100 \mathrm{~mm}^{3}$.

Although, the bulk magnets have a large energy product $(B H)_{\max }$ and high coercivity $\left(H_{c}\right)$, deposition of these materials for microelectromechanical system (MEMS) is a challenge. Sintering or melt spinning is a few of the industrial

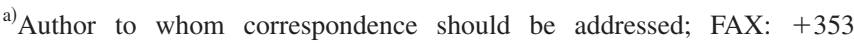

214270271; electronic mail: saibal.roy@ tyndall.ie
}

deposition processes for $\mathrm{NdFeB}, \mathrm{SmCo}$, and other rare-earth magnets, but these processes usually require very high temperatures and are incompatible with MEMS fabrication. ${ }^{8} \mathrm{Al}-$ ternatives to the sintered magnets are hard magnets such as $\mathrm{CoPt}$ and FePt face centered tetragonal (fct) phases. These magnets can be deposited by sputtering ${ }^{9,10}$ or by electroplating. ${ }^{11-14}$ The use of electroplated or sputtered magnets allows for the scaling down of the size of the power generator and also the processes are compatible with MEMS fabrication. Electroplating has the advantage over sputtering of having an inexpensive, faster deposition rate, which readily lends itself to any complex three-dimensional (3D) topology of different devices.

This paper describes the theory, design and simulation of electromagnetic micropower generators with electroplated micromagnets. The volume of the device is approximately $30 \mathrm{~mm}^{3}$ with a predicted maximum power of $85 \mu \mathrm{W}$. In this study, the mechanical and electrical characteristics of two designs of power generator are compared. The twodimensional (2D) finite element analysis (FEA) has been carried out to predict the voltage and power generated by the device using the magnetic properties of electroplated $\mathrm{Co}_{50} \mathrm{Pt}_{50}$ fct phase, with the highest coercivity of $1.3 \mathrm{~T}$ in electroplated films reported in literature, ${ }^{15}$ for a frequency typically present in industrial machinery.

According to Faraday's law, any time change in the magnetic field linking a coil will cause a voltage to be induced in the coil. In the present designs, the magnets are fixed and the coil moves in between two sets of magnets. If it is assumed that the movement of the coil is described by a second order damped mechanical system, with one degree of freedom in the $z$ direction, then the maximum displacement of the coil at the resonant frequency is given by ${ }^{16}$

$$
Z_{\max }=\frac{F}{D 2 \pi f},
$$

where $F$ is the resonant driving force, $D$ is the damping factor, and $f$ is the resonant frequency. $D$ will be composed of electromagnetic damping and air damping. The effect of 


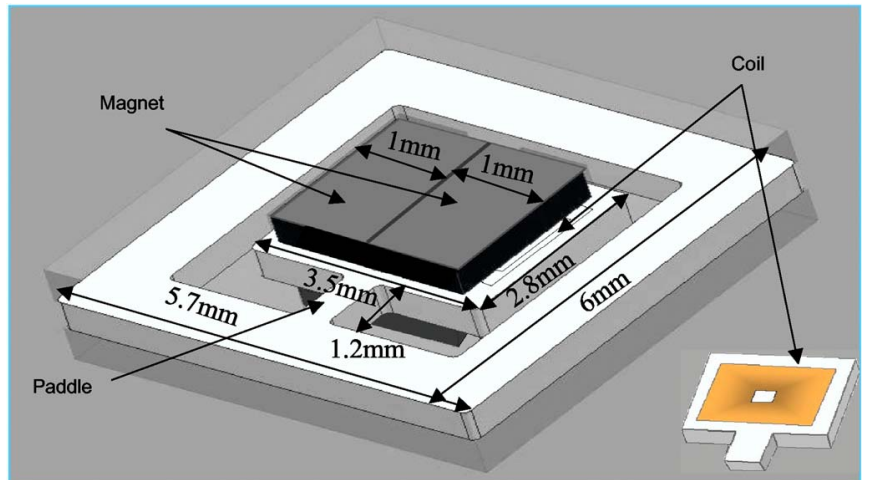

FIG. 1. Schematic of micropower generator. Inset: Si paddle with $\mathrm{Cu}$ coil.

air damping is neglected in this case, which has been explained later. The maximum power generated by the system at resonant frequency is given by

$$
P_{\max }=\frac{F^{2}}{2 D} .
$$

In general the maximum displacement of the generator will be limited by the generator structure and size. Thus, the value of $D$ has to be selected such that the system oscillates at the maximum allowable displacement. The damping factor for a system varies with the gradient of flux linkage and can be written as ${ }^{16}$

$$
D(z)=\left(\frac{d \phi}{d z}\right)^{2} \frac{N^{2}}{R_{\text {coil }}+R_{\text {load }}},
$$

where $d \phi / d z$ is the gradient of flux linkage per turn at a given position, $N$ is the number of turns of the coil, $R_{\text {coil }}$ is the coil resistance, and $R_{\text {load }}$ is the load resistance. Using Eq. (3), the value of the damping factor $D$ can be set to optimize the maximum power generated by a system for a given displacement by changing the load resistance and the number of turns in the coil.

In the present designs, the electromagnetic power generator uses a vibrating two-layer copper coil structure in a magnetic field to generate power. The $10 \mu \mathrm{m}$ thick copper coils are electrodeposited on the top of an insulated $2 \mu \mathrm{m}$ thick bottom copper coil, which is sputtered on a silicon paddle $\left(3.5 \times 2.8 \times 0.5 \mathrm{~mm}^{3}\right)$. The paddle is etched by deep reactive ion etching (DRIE) and batch fabricated on a silicon wafer. A silicon cantilever beam $\left(0.3 \times 1.2 \times 0.5 \mathrm{~mm}^{3}\right)$ connects the paddle to the frame. This coil arrangement is bonded between two sets of $1 \mathrm{~mm}$ wide and $150 \mu \mathrm{m}$ thick $\mathrm{Co}_{50} \mathrm{Pt}_{50}$ fct phase hard magnets, ${ }^{15}$ electroplated on separate silicon wafers (Fig. 1). In the present designs, a $150 \mu \mathrm{m}$ separation was chosen considering the fabrication allowances. On the application of external vibration, the silicon paddle resonates horizontally between the magnets. In design 1 , the magnets are arranged to form a two-pole system with the oppositely polarized magnets placed next to each other (Fig. 2). The positioning of the coils is such that each magnet pole covers one-half of the coil. The advantage of such an arrangement is that while vibrating, the coil moves from a positive flux density to a negative flux density, thereby maximizing a flux gradient and hence generating the voltage. In order to concentrate the magnetic lines of force in the region between the magnets, design 2 uses a $300 \mu$ m thick layer of $\mathrm{Ni}_{45} \mathrm{Fe}_{55}$ soft magnetic (SM) material plated ${ }^{17}$ under the hard

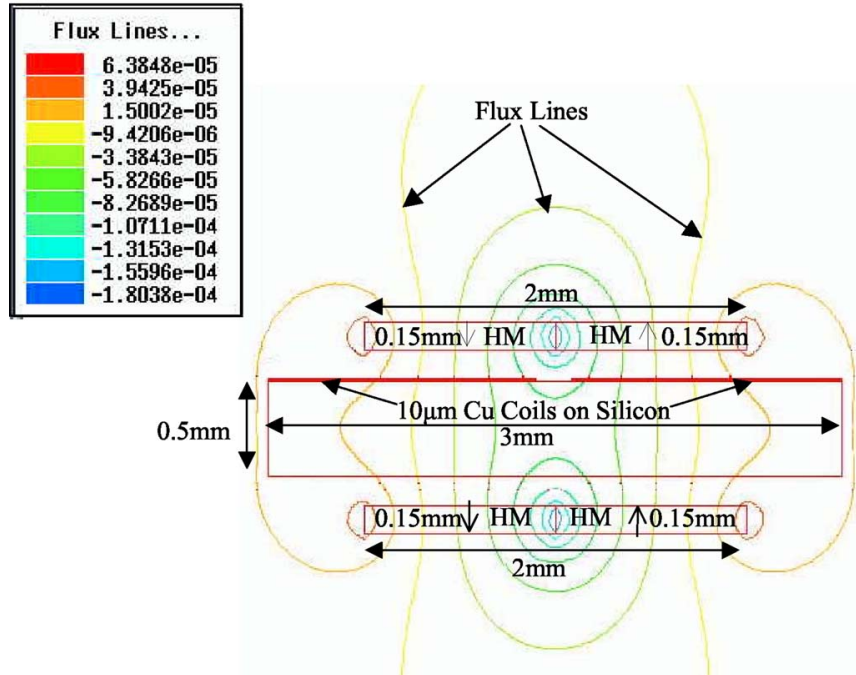

FIG. 2. Flux lines for moving coil on silicon with hard magnets (design 1).

magnet (HM), as shown in Fig. 3. $\mathrm{Ni}_{45} \mathrm{Fe}_{55}$ as a soft material is chosen because of its relatively high flux density (1.6 T) and low coercive field $(1.5 \mathrm{Oe})$ to help concentrate the flux lines. From the simulation results, the flux density present in the majority of the soft magnetic layer is around $1.5 \mathrm{~T}$. Hence, the soft magnetic layer can act as a path for flux lines without exceeding the saturation flux density of the material.

The voltage and power generated by the device are predicted using 2D FEA software. For a maximum power output, the resonant frequency of the electromagnetic power generator should match the frequency of external vibrations. For the simulation, an input velocity given by $v$ $=Z_{\max } 2 \pi f \sin (2 \pi f t)$ defines the motion of the coils in the device. As mentioned earlier, the size and structure of the generator limit the maximum displacement $Z_{\max }$ of the paddle. The value of $Z_{\max }$, for this particular design, is calculated to be $240 \mu \mathrm{m}$. The mass of the moving coil was calculated to be $1.42 \times 10^{-5} \mathrm{~kg}$. The inputs to the $2 \mathrm{D}$ FEA are frequency and acceleration. The values of frequency and acceleration are taken as $7400 \mathrm{~Hz}$ and $1.1 \mathrm{~m} / \mathrm{s}^{2}$. These values were found from measurements taken on a typical industrial fan. The value of the driving force for the given inputs is $1.56 \times 10^{-5} \mathrm{~N}$. According to Zhang et al. ${ }^{18}$ the effect of air damping becomes significant, when the device is scaled down to micrometer dimensions and the working frequency is in the megahertz to gigahertz range. Since in our device the beam which is much larger in dimensions $(1.2 \times 0.3$ $\left.\times 0.5 \mathrm{~mm}^{3}\right)$ vibrates at a lower resonant $(7400 \mathrm{~Hz})$ fre-

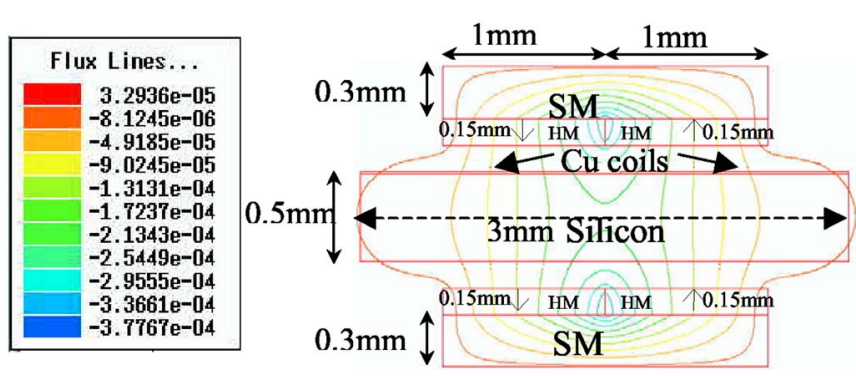

FIG. 3. Flux lines for moving coil on silicon with soft and hard magnets (design 2). 


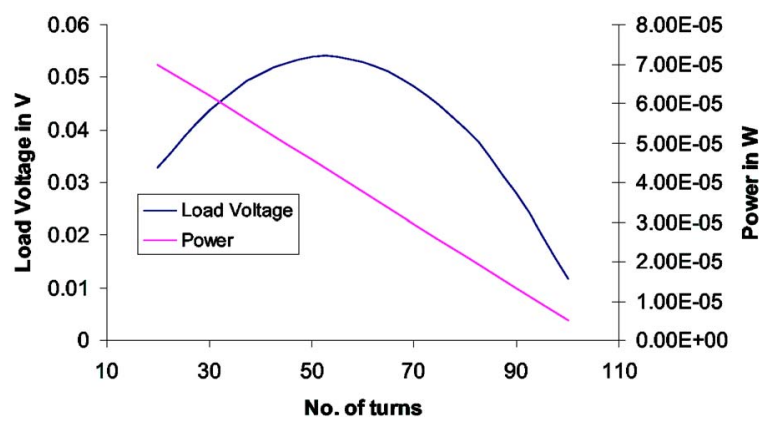

FIG. 4. Variation of load voltage and power with the number of turns for design 1 .

quency, we may assume the effect of air damping as small. This is consistent with the description of air damping effects on silicon resonators studied by Newell ${ }^{19}$ given the size and aspect ratio of the generator. Again the fabricated device could be encapsulated in vacuum, reducing further the effect of air damping. From Eq. (1) it can be calculated that the damping factor be $1.4 \times 10^{-6} \mathrm{~N} \mathrm{~s} / \mathrm{m}$, in order to obtain a $240 \mu \mathrm{m}$ displacement. From FEA, the values of flux gradient per turn for designs 1 and 2 are calculated as $1.84 \times 10^{-4}$ and $4.93 \times 10^{-4} \mathrm{~Wb} / \mathrm{m}$ and the flux densities in the air gap are $0.21 \mathrm{~T}$ and $0.45 \mathrm{~T}$, respectively. The voltage and power generated by the coil with different number of turns are calculated, keeping the damping factor constant for optimized results.

Figures 4 and 5 show how the load power and load voltage vary with the number of turns in the coil for designs 1 and 2. In design 2 the value of flux gradient $(d \Phi / d x)$ is higher, hence the generated voltage, as compared to design 1 due to the presence of soft magnetic layers. For design 1, the maximum voltage of $55 \mathrm{mV}$ is generated with a coil of 60 turns and a load resistance of $37.5 \Omega$. The maximum power for this design is $70 \mu \mathrm{W}$ for a 20 turn coil and a load resistance of $7.75 \Omega$. Similarly, for design 2 , a maximum voltage of $950 \mathrm{mV}$ is generated by a 250 turn coil and a load resistance of $7.5 \mathrm{k} \Omega$ and a maximum power of $85 \mu \mathrm{W}$ is generated with a 20 turn coil and a load resistance of $70 \Omega$. Although, the simulations give the maximum load voltage at 400 turns, the calculations of load voltage and power are carried out up to a maximum 250 turns, because of the limit in the lithographic resolution ( $2.8 \mu \mathrm{m}$ track width) of the electroplated coil. The output voltage is calculated as follow:

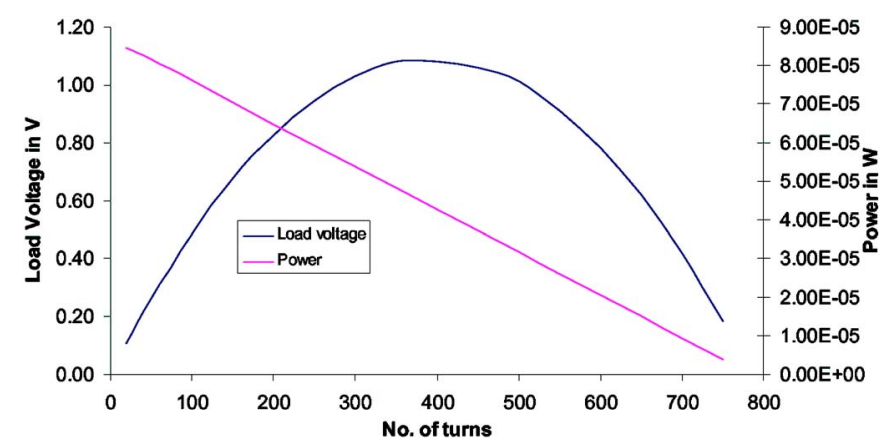

FIG. 5. Variation of load voltage and power with the number of turns for design 2 .

$$
V_{o}=2 \pi N f Z_{\max } \frac{d \phi}{d x} .
$$

Therefore, from Eq. (4) it is clear that an increase in the number of turns leads to an increase in the generated voltage. Similarly the increase in the number of turns leads to an increase in the coil resistance. The value of load resistance has to be varied to keep the damping factor constant, for the beam to resonate at $240 \mu \mathrm{m}$. The load voltage can be calculated as

$$
V_{\text {load }}=V_{o} \frac{R_{\text {load }}}{\left(R_{\text {coil }}+R_{\text {load }}\right)} .
$$

As the generated voltage increases with the number of turns, the load voltage increases until the coil resistance equals the load resistance. After this point, the coil resistance increases exponentially with the number of turns and the load resistance has to be reduced to keep the damping factor constant, leading to a decrease in the load voltage. For power calculations, the variation of the power output with load resistance can be explained using the following equation:

$$
P_{\text {load }}=\frac{V_{\text {load }}^{2}}{2 R_{\text {load }}} \text {. }
$$

This work compares the mechanical and electrical performances of different designs of electromagnetic power generators. The effect of varying the coil turns in order to optimize the output power and load voltage has been investigated. The maximum voltage and power from the first design are $55 \mathrm{mV}$ and $70 \mu \mathrm{W}$, for an input frequency of $7.4 \mathrm{kHz}$ and an acceleration of $1.1 \mathrm{~m} / \mathrm{s}^{2}$ for an optimized choice of load resistance. The maximum displacement of the beam is fixed at $240 \mu \mathrm{m}$. Similarly for the same inputs, the second design generates a maximum voltage of $950 \mathrm{mV}$ and a maximum power of $85 \mu \mathrm{W}$. The power generators are being fabricated using standard MEMS processing techniques, based on the optimized designs. Details of the fabrication processes and measured output of the batch-fabricated devices will be published elsewhere.

The authors would like to acknowledge the financial support by the European Union Framework 6 STREP project VIBES (No. 507911).

${ }^{1}$ A. Chandrakasan et al., Proceedings of the IEEE International Symposium on Circuits and Systems (ISCAS '98), 31 May-3 June, 1998, Vol. 4, pp. 604-607.

${ }^{2}$ W. R. Davis et al., Proceedings of the IEEE Integrated Circuits Conference, CICC'2001, San Diego, CA, May 6-9, 2001, pp. 545-548.

${ }^{3}$ F. Lu et al., Smart Mater. Struct. 13, 57 (2004).

${ }^{4}$ M. Allen, Adv. Mater. Processes 163, 22 (2005).

${ }^{5}$ P. Glynne-Jones et al., Sens. Actuators, A 110, 344 (2004).

${ }^{6}$ E. P. James et al., Sens. Actuators, A 110, 171 (2004).

${ }^{7}$ E. Koukharenko et al., J. Microsyst. Technol. (in press).

${ }^{8}$ S. Guan et al., Sens. Actuators, A 118, 307 (2005).

${ }^{9}$ S. Stavroyiannis et al., Appl. Phys. Lett. 73, 23 (1998).

${ }^{10}$ J. P. Liu et al., Appl. Phys. Lett. 4, 72 (1998).

${ }^{11}$ I. Zana et al., Electrochem. Solid-State Lett. 6, C153 (2003).

${ }^{12}$ V. Georgescu et al., J. Magn. Magn. Mater. 156, 27 (1996).

${ }^{13}$ G. Zangari et al., J. Magn. Magn. Mater. 157-158, 256 (1996).

${ }^{14}$ F. M. F. Rhen et al., IEEE Trans. Magn. 39, 2699 (2003).

${ }^{15}$ F. M. F. Rhen et al., J. Appl. Phys. 97, 113908 (2005).

${ }^{16} \mathrm{C}$. Saha et al., 40th International Universities Power Engineering Conference, Cork, Ireland (UPEC2005), September 7-8, 2005.

${ }^{17}$ S. Roy et al., J. Magn. Magn. Mater. 290-291, 1524 (2005).

${ }^{18}$ C. Zhang et al., Math. Mech. Solids 8, 315 (2003).

${ }^{19}$ W. E. Newell, Science 161, 1320 (1968). 